\title{
Impact of Intraoperative Donor Management on Short-Term Renal Function After Laparoscopic Donor Nephrectomy
}

Eric J. Hazebroek, MD, ${ }^{*}$ Diederik Gommers, MD, PhD, $†$ Michiel A. Schreve, MD, ${ }^{\star}$ Teun van Gelder, MD, PhD, $\ddagger$ Joke I. Roodnat, MD, ‡ Willem Weimar, MD, PhD, $\neq$ H. Jaap Bonjer, MD, PhD, ${ }^{\star}$ and Jan N. M. IJzermans, MD, PhD*

From the Departments of *Surgery, †Anesthesiology, and łInternal Medicine, University Hospital Rotterdam-Dijkzigt, the Netherlands

\section{Objective}

To determine whether intraoperative diuresis, postoperative recovery, and early graft function differ between laparoscopic open nephrectomy (LDN) and open donor nephrectomy (ODN).

\section{Summary Background Data}

Laparoscopic donor nephrectomy can reduce donor complications in terms of decreased pain and shorter convalescence. Although its technical feasibility has been established, concerns have been raised about the impaired renal function resulting from pneumoperitoneum and short- and long-term function of kidneys removed by LDN.

\section{Methods}

Between December 1997 and December 2000, 89 LDNs were performed at the authors' institution. These were compared with 83 conventional ODNs performed between January 1994 and December 1997. Graft function, intraoperative variables, and clinical outcome were compared.

\section{Results}

Laparoscopic donor nephrectomy was attempted in 89 patients and completed in 91\% (81/89). Length of hospital stay was significantly shorter in the laparoscopic group. During kidney dissection, the amount of fluids administered and intraoperative diuresis were significantly lower for LDN. In recipients, mean serum creatinine was higher after LDN compared with ODN 1 day after surgery. From postoperative days 2 until 28 , there were no differences in serum creatinine. Graft survival rates were similar for LDN and ODN.

\section{Conclusions}

Donors can benefit from an improvement in postoperative recovery after LDN. Assessment of an adequate perioperative hydration protocol is mandatory to ensure optimal kidney quality during laparoscopic procurement. The initial graft survival and function rates justify continued development and adoption of LDN.
Laparoscopic donor nephrectomy (LDN) has been developed to reduce postoperative pain, shorten convalescence, and improve the cosmetic outcome of the kidney donor. LDN has the potential to increase the number of kidney donations by removing some of the disincentives inherent to donation. ${ }^{1}$ Since the initial report by Ratner et al. ${ }^{2}$ in 1995, LDN has been adopted by a number of institutions world-

\footnotetext{
Correspondence: Jan N. M. IJzermans, MD, PhD, Department of Surgery, University Hospital Rotterdam-Dijkzigt, Dr. Molewaterplein 40, 3015 GD Rotterdam, the Netherlands.

E-mail: ijzermans@hlkd.azr.nl

Accepted for publication November 7, 2001.
}

DOI: 10.1097/01.SLA.0000018663.03354.12 wide. However, concern has been raised about the reported incidence of primary dysfunction of transplanted kidneys after laparoscopic procurement. ${ }^{3}$ Although retrospective studies comparing LDN with the conventional approach suggest similar graft function after 1 year, it has been shown that recipients of laparoscopically procured kidneys have higher serum creatinine levels and a greater need for dialysis in the first weeks after transplantation. ${ }^{3-5}$ Mechanical injury to the graft, longer warm ischemia time, and pneumoperitoneum have all been suggested as causative factors. Clinical and experimental studies have shown that increased intraabdominal pressure can cause transient renal dysfunction (oliguria) as a result of impaired renal blood flow, caused by compression of the renal parenchyma and renal 
vein. $^{6-8}$ In view of these data, use of the laparoscopic approach as an alternative to the gold standard of open nephrectomy can be justified only if it is clear that allograft function after laparoscopic kidney procurement is not at stake. At present, no randomized controlled clinical trials are at hand, and the follow-up of most series has been relatively short. Further, the current lack of an adequate evidence base for LDN obligates institutions performing this technique to report on safety and effectiveness after engraftment. ${ }^{9}$

This article compares our 3-year experience with LDN and that of a control cohort of patients undergoing open donor nephrectomy (ODN) to determine whether early graft function, intraoperative diuresis, and postoperative recovery differ between LDN and ODN.

\section{METHODS}

\section{Patient Selection}

Laparoscopic donor nephrectomy was performed in 89 patients from December 1997 through December 2000. These laparoscopic donors were compared with a cohort of 83 patients undergoing ODN at our institution from January 1994 through December 1997. After the introduction of the laparoscopic technique in December 1997, ODN was performed in six patients, but these were excluded from analysis. The reasons for performing the open technique were either obesity $(n=4)$ or multiple renal arteries $(n=2)$. Patient data were collected from medical records and were compared for age, gender, body mass index, and comorbidity. Operative and postoperative data collected included blood loss, warm ischemia time, length of operation, length of postoperative hospital stay, and complications. Complications were defined as events within the perioperative period that altered patient recovery, prolonged hospital stay, or technically changed the surgical procedure. Pre- and postoperative serum creatinine clearance values were calculated using the Cockcroft-Gault formula ${ }^{10}$ and were compared between ODN and LDN. During surgery, the amount of intravenous hydration fluids and osmotic diuretics administered and urine output in the donor until the moment of nephrectomy were compared. Graft function and survival were compared for the two groups. Delayed graft function was defined as the need for dialysis in the postoperative period. Quantification of urine production after engraftment was documented in both groups. Mean serum creatinine levels at $1,2,3,4,5,7,14$, and 28 days after transplantation were compared for all recipients. Fluid intake and diuresis from the moment of transplantation until 10 hours after surgery were compared for all recipients. Four living donor transplants with pediatric recipients (younger than 16 years) were excluded from the study.

Candidates for donor nephrectomy were screened thoroughly by medical history, physical examination, blood and urine chemistry, and immunologic and infectious disease studies. Standard preoperative screening included renography, Seldinger angiography, and selective renal artery angiography in patients with more than one renal artery. Ultrasonography was performed to exclude the presence of kidney deformities. It was decided before surgery from which side the kidney would be procured. If both kidneys had normal function and normal anatomy, the right kidney was preferred for LDN because on this side the gonadal and adrenal veins do not originate from the right renal vein.

\section{Operative Technique}

All ODNs were performed by the same transplant surgeon. In the first 30 procedures, LDN was performed by the transplant surgeon and a general surgeon with advanced laparoscopic training. After 30 procedures, LDN was performed by the transplant surgeon, assisted by one of the surgical residents.

All ODNs were performed through a retroperitoneal flank incision, without partial rib resection. LDN was performed under general endotracheal anesthesia with the patient in semilateral decubitus position. The operating table was flexed maximally to expose the space between the iliac crest and the costal margin. Positioning of the patient allowed, if necessary, conversion to ODN by standard lumbotomy. Orogastric suction and bladder catheterization were used routinely. Antibiotics were not routinely administered.

A $30^{\circ}$ laparoscope was introduced through a Hasson trocar, placed through a small midline incision, just caudally to the umbilicus. At the end of the procedure this incision was extended to 5 to $6 \mathrm{~cm}$ to enable extraction of the kidney. A pneumoperitoneum of no more than $12 \mathrm{~mm} \mathrm{Hg}$ was created, and four additional trocars were inserted. One $10-\mathrm{mm}$ trocar port was placed at the lateral margin of the rectus muscle, equidistant to the umbilicus and the superior iliac spine. The second $10-\mathrm{mm}$ trocar was placed laterally between the costal margin and the iliac crest. Two 5-mm ports were placed in the midline between the xiphoid process and the umbilicus. The more cephalad of these two ports was used to insert a small endo-Babcock clamp for retraction of the liver (right nephrectomy) or the spleen (left nephrectomy) by fixing it to the lateral abdominal wall.

The operation for right nephrectomy was conducted as follows: mobilization of the right colon using an ultrasonic device (Ultracision, Ethicon, Somerville, NJ), opening of the renal fascia, and division of the renal fat. The renal vein was dissected up to its entrance in the caval vein and encircled with a rubber vessel-loop to enable gentle traction and correct positioning of the stapling device. Attention was given to occasional lumbar veins at the confluence of the renal and caval veins. In case of multiple renal arteries, medial rotation of the kidney enabled a dorsal view and access to the hilar structures. Left donor nephrectomy was conducted in a similar fashion: mobilization of the left colon and spleen, dissection of the renal vein up to its crossing with the aorta, dissection of the renal artery, ligation of 
adrenal and ovarian or spermatic vein with titanium clips, dissection of the ureter, creation of an extraction incision, anticoagulation, division of the ureter, renal artery, and renal vein, and extraction of the kidney.

After administering 5,000 U heparin systemically, the ureter, renal artery, and renal vein were divided using a linear vascular laparoscopic stapler (EndoGIA 30, U.S. Surgical, Norwalk, CT). A plastic extraction bag (Endocatch, U.S. Surgical) was used to extract the kidney through the subumbilical incision. Directly after kidney extraction, hemostasis was restored by protamine sulfate. The kidney was perfused with Euro-Collins solution at $4^{\circ} \mathrm{C}$ and stored on ice awaiting transplantation. After closure of the extraction incision, pneumoperitoneum was reestablished and the operative field inspected. After adequate hemostasis was ensured, ports were removed under direct visualization, the abdomen was desufflated, and incisions were closed.

\section{Statistical Analysis}

Statistical analysis was performed using the SPSS 9.0 (SPSS Inc., Chicago, IL) statistical software package. Comparisons between ODN and LDN were performed using the Mann-Whitney test. Categorical data were reported as absolute number of patients and/or the percentage of the group studied and were compared using $2 \times 2$ contingency tables and chi-square tests. Adjustments for multiple covariates were made using linear regression for continuous outcomes. Survival analyses were performed using Kaplan-Meier techniques, compared with log-rank tests. Statistical significance was accepted at $P<.05$.

\section{RESULTS}

Patient demographics and operative data are shown in Table 1. LDN was attempted in 89 patients and completed in $81(91 \%)$. In six patients, a pneumatic sleeve (Omniport, ASC, Bray, Ireland) was used to allow performance of hand-assisted nephrectomy to control vascular bleeding or to facilitate dissection. Eight patients required conversion to flank laparotomy. In one patient undergoing left LDN, laceration of the splenic capsule required lumbotomy and subsequent splenectomy. Six conversions occurred after vascular injuries to either the lumbar vein or the renal vein. Although blood loss was limited in these patients, the technical difficulty in repairing these lesions laparoscopically and the desire to prevent damage to the kidney from prolonged ischemia warranted conversion to lumbotomy. In one patient, bleeding from a trocar site resulting from a lesion from the epigastric vessels necessitated conversion after kidney extraction.

Postoperative complications after LDN and ODN are listed in Table 2. Both techniques had low complication rates, and the number of patients with complications was not different. One patient in the ODN group died 6 days after an
Table 1. PATIENT DEMOGRAPHICS AND OPERATIVE DATA

\begin{tabular}{lccc}
\hline & $\begin{array}{c}\text { LDN } \\
\text { (n = 89) }\end{array}$ & $\begin{array}{c}\text { ODN } \\
(\mathbf{n}=\mathbf{8 3})\end{array}$ & $\boldsymbol{P}$ Value \\
\hline Age (yr) (mean + range) & $46.9(20-76)$ & $47.1(20-77)$ & $\mathrm{NS}$ \\
Sex & & & \\
$\quad$ Male & $48(54 \%)$ & $34(41 \%)$ & $\mathrm{NS}$ \\
$\quad$ Female & $41(46 \%)$ & $49(59 \%)$ & $\mathrm{NS}$ \\
Body mass index & $25.4(17-35)$ & $25.5(16-36)$ & $\mathrm{NS}$ \\
$\quad$ (mean + range) & & & \\
ASA class & & & \\
$\quad 1$ & $73(82 \%)$ & $68(82 \%)$ & $\mathrm{NS}$ \\
2 & $16(18 \%)$ & $15(18 \%)$ & $\mathrm{NS}$ \\
Origin & & & \\
$\quad$ Living-related & $69(78 \%)$ & $77(93 \%)$ & $<.001$ \\
$\quad$ Living-unrelated & $20(22 \%)$ & $6(7 \%)$ & $<.001$ \\
Side & & & \\
$\quad$ Left & $26(29 \%)$ & $47(57 \%)$ & .002 \\
$\quad$ Right & $63(71 \%)$ & $36(43 \%)$ & .002 \\
Conversion & $8(8.9 \%)$ & &
\end{tabular}

LDN, Laparoscopic donor nephrectomy; ODN, open donor nephrectomy; ASA, American Society of Anesthesiologists.

uneventful donation procedure as a result of cardiac ischemia.

Perioperative data for ODN and LDN are shown in Table 3. Overall, mean operative time from skin incision to closure was longer for LDN (235 vs. 155 minutes). Mean intraoperative blood loss was comparable between both groups. Length of hospital stay was significantly shorter in the LDN group (3.9 vs. 6.2 days). The reduction in creatinine clearance 2 days after nephrectomy was more pronounced in the laparoscopic group.

Intraoperative hydration and diuresis during kidney dissection were documented until extraction of the graft (Table 4). Patients undergoing ODN had a significantly higher

Table 2. POSTOPERATIVE COMPLICATIONS IN KIDNEY DONORS

\begin{tabular}{llll}
\hline \multicolumn{1}{c}{ LDN } & & \multicolumn{2}{c}{ ODN } \\
\hline $\begin{array}{l}\text { Hemorrhage, conservative } \\
\quad \text { treatment }\end{array}$ & 1 & Hematoma & 1 \\
$\begin{array}{l}\text { Hemorrhage small laparotomy } \\
\quad \text { wound, reoperation }\end{array}$ & 1 & Urinary tract infection & 2 \\
Subcutaneous emphysema & 1 & Infection epidural catheter & 1 \\
Fever & 2 & Fever & 4 \\
Urinary tract infection & 2 & Exanthema after epidural & 1 \\
Bronchitis & 1 & Incisional hernia & 2 \\
Pneumonia & 2 & Bowel perforation & 1 \\
Number of patients with $>1$ & 1 & & 2 \\
$\quad$ complication & & & 1 \\
Death & 0 & &
\end{tabular}

LDN, Laparoscopic donor nephrectomy; ODN, open donor nephrectomy. 
Table 3. PERIOPERATIVE DATA IN KIDNEY DONORS

\begin{tabular}{|c|c|c|c|}
\hline & LDN & ODN & $P$ Value \\
\hline Length of operation $(\mathrm{min})^{*}$ & $235(105-420)$ & $155(75-310)$ & $<.001$ \\
\hline Estimated blood loss ( $\mathrm{mL}$ ) & 375 (50-2,300†) & $352(100-1,000)$ & NS \\
\hline Hospital stay (days) & $3.9(2-9)$ & $6.2(3-31 \neq)$ & $<.001$ \\
\hline Creatinine clearance, preop $(\mathrm{mL} / \mathrm{min}) \S$ & $104(55-179)$ & $109(50-197)$ & NS \\
\hline Creatinine clearance, 2 days postop $(\mathrm{mL} / \mathrm{min}) \S$ & $72(41-117)$ & $82(40-132)$ & .011 \\
\hline \multicolumn{4}{|c|}{$\begin{array}{l}\text { Data are given as mean (range). LDN, laparoscopic donor nephrectomy; ODN, open donor nephrectomy. } \\
\text { * Time from skin incision to closure } \\
\text { † One patient required blood transfusion due to a lesion from the epigastric vessels. } \\
\text { † Patient requiring reoperation due to bowel perforation; stay was } 31 \text { days. } \\
\S \text { Calculated using the Cockcroft-Gault formula. }\end{array}$} \\
\hline
\end{tabular}

amount of fluids administered (colloids and crystalloids) during kidney dissection (8.1 and 22.4 vs. 3.4 and 16.2 $\mathrm{mL} / \mathrm{kg} / \mathrm{h}$, respectively). Comparison of intraoperative urine output until the moment of nephrectomy showed that this was significantly lower for the laparoscopic group (1.6 vs. $2.8 \mathrm{~mL} / \mathrm{kg} / \mathrm{h}$ ). Information on medication administered to promote diuresis was available in 86 LDNs and 69 ODNs. In $47 \%$ of LDNs, osmotic diuretics or dopamine was administered. Warm ischemia times were significantly longer in the LDN group (7.8 vs. 4.8 minutes).

Graft function and survival are compared in Table 5. Information on intraoperative urine production of the kidney during engraftment was documented in the operative charts of 62 LDNs and 78 ODNs. There was a significant difference between LDN and ODN grafts that were producing urine subsequent to reperfusion $(73 \%$ vs. $88 \%)$. Fluid intake of recipients from the moment of transplantation until 10 hours after surgery was similar in both groups. During this period urine production was lower in recipients of a kidney removed by LDN (2.9 vs. $3.9 \mathrm{~L}, P=.004)$.

Mean serum creatinine levels in the first month after transplantation are also shown in Table 5. Before surgery, there were no differences in serum creatinine levels between LDN and ODN patients. On postoperative day 1, the mean serum creatinine level was significantly higher in the laparoscopic group (covariate analysis). From day 2 on, the differences in mean serum creatinine levels between LDN and ODN were no longer significant.
Duration of follow-up was longer for ODN patients because these procedures were performed in the years before the introduction of LDN. Graft survival has been maintained in $86(97 \%)$ of 89 transplanted kidneys after LDN with a mean follow-up of 18.3 months (range 1-37). Graft failure resulting from arterial thrombosis occurred in one patient, 1 day after uneventful laparoscopic harvest, necessitating transplant nephrectomy. We observed delayed graft function in three patients in the LDN group, requiring posttransplant dialysis in the first week.

\section{DISCUSSION}

There are several concerns with the application of the laparoscopic approach for live renal donation. Live donor nephrectomy involves a healthy individual who is subjected to major surgery for the benefit of another individual. At all times, the operation must be safe. Further, laparoscopic procurement of a kidney should provide excellent recipient graft outcomes. Several studies have reported that LDN can be performed safely in selected candidates with a reduction in postoperative complications and shorter hospital stays compared with ODN. ${ }^{4,11-14}$

Laparoscopic kidney removal does take more time than the conventional approach. However, it has been shown that operative times of LDN decrease with increased experience. ${ }^{14}$ Although most studies comparing LDN with the conventional approach suggest similar graft function after 1

Table 4. INTRAOPERATIVE DATA IN KIDNEY DONORS

\begin{tabular}{|c|c|c|c|}
\hline & LDN & ODN & $P$ Value \\
\hline \multicolumn{4}{|l|}{ IV fluid hydration } \\
\hline Colloid (mL/kg/h) & $3.4(0-11.7)$ & $8.1(0-92.7)$ & $<.001$ \\
\hline Crystalloid (mL/kg/h) & $16.2(4.3-51.4)$ & $22.4(2.0-45.5)$ & $<.001$ \\
\hline Administration of diuretics* or dopamine (number of cases) & $40 / 86(47 \%)$ & $27 / 69(39 \%)$ & NS \\
\hline Urine output until nephrectomy $(\mathrm{mL} / \mathrm{kg} / \mathrm{h})$ & $1.6(0.3-6.1)$ & $2.8(0.4-11.8)$ & $<.001$ \\
\hline Warm ischemia time (min) & $7.8(2-17)$ & $4.8(2-12)$ & $<.001$ \\
\hline
\end{tabular}

Data are given as mean (range). LDN, laparoscopic donor nephrectomy; ODN, open donor nephrectomy. * Mannitol and/or furosemide. 
Table 5. GRAFT FUNCTION AND SURVIVAL

\begin{tabular}{|c|c|c|c|}
\hline & LDN & ODN & $P$ Value \\
\hline Immediate urine production & 45/62 (73\%) & 69/78 (88\%) & .016 \\
\hline Fluid intake until 10 hrs postop $(L)^{\star}$ & $4.9 \pm 0.17$ & $4.7 \pm 0.15$ & NS \\
\hline Urine production until $10 \mathrm{hrs}$ postop $(\mathrm{L})^{\star}$ & $2.9 \pm 0.17$ & $3.9 \pm 0.23$ & .004 \\
\hline \multicolumn{4}{|l|}{ Mean serum creatinine $(\mu \mathrm{mol} / L)^{*}$} \\
\hline Preop & $819 \pm 36.6$ & $782 \pm 33.9$ & NS \\
\hline Postop day 1 & $416 \pm 26.5$ & $344 \pm 21.5$ & .043 \\
\hline Postop day 2 & $227 \pm 23.5$ & $182 \pm 14.9$ & NS \\
\hline Postop day 3 & $195 \pm 23.6$ & $147 \pm 12.0$ & NS \\
\hline Postop day 4 & $199 \pm 23.9$ & $142 \pm 11.3$ & NS \\
\hline Postop day 5 & $200 \pm 22.9$ & $146 \pm 13.3$ & NS \\
\hline 1 week & $197 \pm 23.5$ & $157 \pm 16.4$ & NS \\
\hline 2 weeks & $155 \pm 14.8$ & $138 \pm 12.9$ & NS \\
\hline 4 weeks & $136 \pm 9.1$ & $127 \pm 9.2$ & NS \\
\hline Delayed graft function & $3 / 89(3.4 \%)$ & 3/83 (3.6\%) & NS \\
\hline One-year graft survival & $86 / 89(97 \%)$ & $76 / 83(92 \%)$ & NS \\
\hline One-year patient survival & 86/89 (97\%) & 77/83 (93\%) & NS \\
\hline
\end{tabular}

LDN, laparoscopic donor nephrectomy; ODN, open donor nephrectomy.

* Data expressed as mean \pm standard error of mean.

year, concerns have been raised about the reported incidence of primary dysfunction after laparoscopic kidney procurement. $^{3}$

The impaired postoperative short-term function of LDN kidney transplants may be due to diminished intraoperative blood flow associated with the pneumoperitoneum, traumatic removal of the kidney graft through a small incision, and longer warm ischemia time. ${ }^{3}$ Clinical and experimental studies have shown that increased intraabdominal pressure can lead to transient renal dysfunction (oliguria) by reducing renal blood flow, caused by compression of the renal parenchyma and renal vein. ${ }^{7,8,15,16}$ However, the effects of procurement of a renal allograft in the altered physiologic environment of pneumoperitoneum are not fully understood. One of the aims of the present study was to retrospectively evaluate perioperative intraoperative fluid requirements for LDN and ODN. Analysis of the operative charts of all kidney donors between 1994 and 2000 revealed that during LDN, a significantly lower amount of fluid was administered during kidney dissection, and that urine production until the moment of kidney extraction was lower compared with the conventional approach. The importance of maintaining urine output at approximately $300 \mathrm{~mL} / \mathrm{h}$ during LDN has been stressed; ${ }^{13}$ however, details on intraoperative diuresis during LDN are seldom reported. In our series, only $47 \%$ of patients undergoing LDN received mannitol or dopamine to encourage renal perfusion. At the initiation of this study, it was our impression that some recipients of a laparoscopically procured kidney do not have immediate function after reperfusion. Analysis of recipients' operative charts showed that after engraftment, confirmation of a brisk diuresis after declamping the renal artery was reported in $88 \%$ after ODN and in $73 \%$ after LDN. Although there was no difference in the amount of fluid administered from start of the transplantation procedure until 10 hours after surgery, during this period urine production was significantly lower in LDN recipients. This difference in postoperative graft function is reflected in a higher mean serum creatinine level on postoperative day 1 after LDN. However, from day 2 onwards, the differences in mean serum creatinine levels between LDN and ODN were no longer significant. These findings suggest that LDN grafts have a slower initial function compared with ODN, but there is no difference in longer-term renal function. In addition, there have been no differences in the incidence of delayed graft function or graft survival between the two groups.

Mean warm ischemia time after LDN in this study was 7.8 minutes, which is longer than reported in other studies. ${ }^{4}$ Despite a longer warm ischemia time, there were no differences in delayed graft function between LDN and ODN. It is not clear what constitutes an acceptable limit, but with increased experience warm ischemia time can be reduced. ${ }^{4}$

Unlike other groups, we performed a substantial number of right-sided LDNs, which is often considered to be one of the drawbacks of the laparoscopic approach. ${ }^{13,17}$ In all right-sided LDNs performed, an adequate length of the renal vein could be obtained. In addition, no problems occurred when performing the venous anastomosis.

In eight patients, the laparoscopic procedure was converted to ODN and subsequently completed. In our opinion, adopting a low threshold to convert to an open procedure is necessary to ensure procurement of the kidney graft in pristine condition with minimal risk for the donor.

Previously, it has been stressed that it will take years before data on long-term graft function after LDN are available. ${ }^{9}$ Therefore, the current lack of an adequate evidence base for LDN obligates institutions performing this 
technique to report on safety and effectiveness after engraftment. ${ }^{9}$ The present study shows our 3-year experience with LDN. In general, development of a successful living donor program requires a dedicated, coordinated multidisciplinary approach. Operating times of LDN are longer, so hydration protocols should be adjusted accordingly. The disparity in intraoperative fluid requirements and diuresis as reported in this study effected improvements in our anesthetic protocol for patients undergoing LDN. In an attempt to ameliorate the decrease in venous return resulting from increased intraabdominal pressure, vigorous hydration is now used during surgery to promote adequate diuresis. ${ }^{18,19}$ Preoperative fluid administration to the donor may be another measure to improve hemodynamics in the kidney. ${ }^{4}$ We have started a prospective randomized clinical trial comparing LDN with ODN that will allow a more valid comparison of recuperation, complications, and graft function.

In conclusion, LDN is a technically feasible but demanding procedure that can be performed with death and complication rates comparable to those of ODN. Donors can benefit from an improvement in postoperative recovery. Although initial graft function and survival rates after LDN are good, long-term follow-up is needed. Special care should be given to intraoperative fluid administration, and anesthesiologists should be trained in the hemodynamic consequences of a pneumoperitoneum during LDN.

\section{Acknowledgments}

The authors thank E. W. Steyerberg, PhD, Department of Public Health, for advice on the statistical analysis and Mrs. J. G. van Duuren-van Pelt for valuable assistance in data management.

\section{References}

1. Ratner LE, Hiller J, Sroka M, et al. Laparoscopic live donor nephrectomy removes disincentives to live donation. Transplant Proc 1997; 29:3402-3403.
2. Ratner LE, Ciseck LJ, Moore RG, et al. Laparoscopic live donor nephrectomy. Transplantation 1995; 60:1047-1049.

3. Nogueira JM, Cangro CB, Fink JC, et al. A comparison of recipient renal outcomes with laparoscopic versus open live donor nephrectomy. Transplantation 1999; 67:722-728.

4. Flowers JL, Jacobs S, Cho E, et al. Comparison of open and laparoscopic live donor nephrectomy. Ann Surg 1997; 226:483-489.

5. Ratner LE, Montgomery RA, Maley WR, et al. Laparoscopic live donor nephrectomy: the recipient. Transplantation 2000; 69):23192323.

6. McDougall EM, Monk TG, Wolf JS, Jr., et al. The effect of prolonged pneumoperitoneum on renal function in an animal model. J Am Coll Surg 1996; 182:317-328.

7. Kirsch AJ, Hensle TW, Chang DT, et al. Renal effects of $\mathrm{CO}_{2}$ insufflation: oliguria and acute renal dysfunction in a rat pneumoperitoneum model. Urology 1994; 43:453-459.

8. Chang DT, Kirsch AJ, Sawczuk IS. Oliguria during laparoscopic surgery. J Endourol 1994; 8:349-352.

9. Merlin TL, Scott DF, Rao MM, et al. The safety and efficacy of laparoscopic live donor nephrectomy: a systematic review. Transplantation 2000; 70:1659-1666.

10. Cockcroft DW, Gault MH. Prediction of creatinine clearance from serum creatinine. Nephron 1976; 16:31-41.

11. Ratner LE, Buell JF, Kuo PC. Laparoscopic donor nephrectomy: pro. Transplantation 2000; 70:1544-1546.

12. Leventhal JR, Deeik RK, Joehl RJ, et al. Laparoscopic live donor nephrectomy-is it safe? Transplantation 2000; 70:602-606.

13. Sasaki TM, Finelli F, Bugarin E, et al. Is laparoscopic donor nephrectomy the new criterion standard? Arch Surg 2000; 135:943-947.

14. Berends FJ, den Hoed PT, Bonjer HJ, et al. Technical considerations and pitfalls in laparoscopic live donor nephrectomy. Surg Endosc (in press).

15. Melville RJ, Frizis HI, Forsling ML, et al. The stimulus for vasopressin release during laparoscopy. Surg Gynecol Obstet 1985; 161:253-256.

16. Richards WO, Scovill W, Shin B, et al. Acute renal failure associated with increased intra-abdominal pressure. Ann Surg 1983; 197:183187.

17. Barry JM. Laparoscopic donor nephrectomy: con. Transplantation 2000; 70:1546-1548.

18. London ET, Ho HS, Neuhaus AM, et al. Effect of intravascular volume expansion on renal function during prolonged $\mathrm{CO}_{2}$ pneumoperitoneum. Ann Surg 2000; 231:195-201.

19. Kavoussi LR. Laparoscopic donor nephrectomy. Kidney Int 2000; 57:2175-2186. 